\title{
Interferometric radius and limb darkening of the asteroseismic red giant $\eta$ Serpentis with the CHARA Array (Research Note)
}

\author{
A. Mérand ${ }^{1, \star}$, P. Kervella ${ }^{2}$, C. Barban ${ }^{2}$, E. Josselin ${ }^{3}$, T. A. ten Brummelaar ${ }^{4}$, H. A. McAlister ${ }^{4}$, V. Coudé du Foresto ${ }^{2}$,
} S. T. Ridgway ${ }^{5}$, N. Turner ${ }^{4}$, J. Sturmann ${ }^{4}$, L. Sturmann ${ }^{4}$, P. J. Goldfinger ${ }^{4}$, and C. Farrington ${ }^{4}$

1 European Organisation for Astronomical Research in the Southern Hemisphere (ESO) Alonso de Córdova 3107, Casilla 19001, Santiago 19, Chile e-mail: Antoine.Merand@eso.org

2 LESIA, Observatoire de Paris, CNRS UMR 8109, UPMC, Université Paris Diderot, 5 Place Jules Janssen, 92195 Meudon, France

3 Groupe de Recherche en Astronomie et Astrophysique du Languedoc (GRAAL), Université Montpellier II, CNRS UMR 5024, 34095 Montpellier Cedex 05, France

4 Center for High Angular Resolution Astronomy, Georgia State University, PO Box 3965, Atlanta, Georgia 30302-3965, USA

5 National Optical Astronomy Observatories, 950 North Cherry Avenue, Tucson, AZ 85719, USA

Received 18 March 2009 / Accepted 4 May 2010

\section{ABSTRACT}

\begin{abstract}
Context. The radius of a star is a very important constraint to evolutionary models, particularly when combined with asteroseismology. Diameters can now be measured interferometrically with great precision (better than 1\%), but the center-to-limb darkening (CLD) remains a potential source of bias. Measuring this bias is possible by completely resolving the star using long-baseline interferometry, and has only been achieved for a handful of stars.

Aims. The red giant $\eta$ Ser (K0III-IV) is a particularly interesting target, as asteroseismic oscillations have recently been detected in this star by spectroscopy. We aim to measure its radius with high accuracy, debiased from limb darkening, in order to bring new constraints to its models.

Methods. We obtained interferometric observations of $\eta$ Ser in the near-infrared using the CHARA/FLUOR instrument, in particular in the so-called second lobe of visibility in order to constrain the CLD and debias our diameter estimation.

Results. The limb darkened angular diameter of $\eta$ Ser is $2.944 \pm 0.010$ mas (using spherical photosphere models PHOENIX and MARCS for the limb darkening), that converts into a radius of $5.897 \pm 0.028 R_{\odot}$ with the Hipparcos parallax. Thanks to a precise visibility measurement in the second lobe of the visibility function of $\eta$ Ser and a one-parameter limb-darkened visibility profile, we were able to show that the photosphere models have the best agreement possible.

Conclusions. Our limb darkening measurement of $\eta$ Ser is in agreement with existing atmosphere models of this star, with a slightly better agreement for models using spherical geometry. This is a strong indication that interferometric angular diameter measurements for red giants, corrected for the CLD using models, are unbiased at a very small level (a fraction of 1\%). In particular, this strengthens our confidence in the existing catalogues of calibrator stars for interferometry that are based on giant stars similar to $\eta$ Ser. The high accuracy of our measurement brings a new and strong constraint for the asteroseismic modeling of this star.
\end{abstract}

Key words. stars: individual: $\eta$ Serp - stars: evolution - stars: fundamental parameters - techniques: interferometric stars: oscillations

\section{Introduction}

It is well known that direct measurements of stellar radii are a powerful tool in discriminating between different numerical stellar models (see for example 2007 and 2007), particularly when combined with asteroseismology. In this context, we present in Sect. 2 a new interferometric measurement of the angular diameter of $\eta$ Ser. This star has recently been the subject of an asteroseismic investigation showing oscillations with a period of $\approx 2.1 \mathrm{~h}$ (Barban et al. 2004; and Hekker et al. 2006, for references). The reported interferometric measurements will provide useful constraints for future modeling efforts on this star. In the following work care was taken to ensure that the high precision result $(0.3 \%)$ was not affected by biases, in particular any bias

^ Formerly a research associate at the CHARA Array, during which period most of the research presented here has been carried out. due to the correction for limb darkening. Using measurements obtained in the second lobe of visibility, we estimate the exponent of the power-law limb darkening model of this star and, in Sect. 4, we compare the predictions of several existing model atmospheres with the observed limb darkening of $\eta$ Ser.

\section{Interferometric observations}

We observed $\eta$ Ser (HD 168723, HR 6869, HIP 89962) in July 2006 at the CHARA Array (ten Brummelaar et al. 2005) using FLUOR, the Fiber Linked Unit for Optical Recombination (Coudé du Foresto et al. 2003; Mérand et al. 2006a). This instrument is equipped with a near infrared $K^{\prime}$ band filter $(1.9 \leq \lambda \leq$ $2.3 \mu \mathrm{m})$.

We extracted the visibilities from the raw data using the FLUOR data reduction software (Coudé du Foresto et al. 1997; 


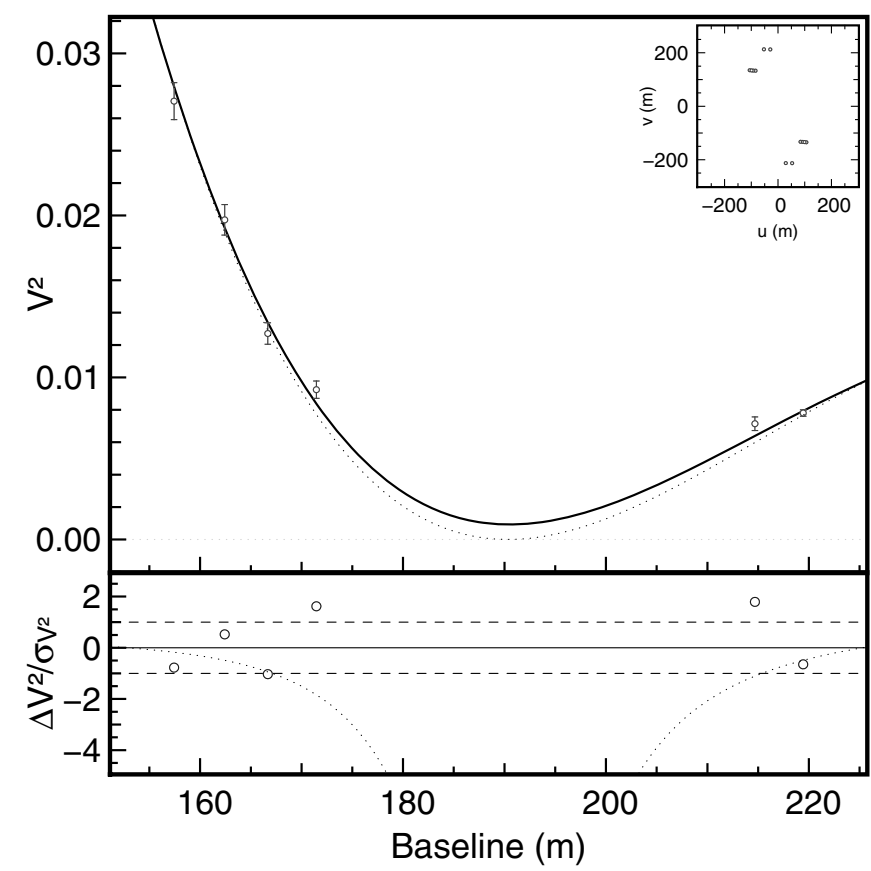

Fig. 1. Squared visibilities and adjusted two-parameter limb darkened disk visibility model of $\eta$ Ser (with bandwidth smearing). The dotted line is the monochromatic visibility curve for $\lambda=2.16$ microns. The lower panel shows the normalized residuals, as well as the difference between the monochromatic visibility profile and the one using bandwidth smearing (dotted line, with a typical error bar of 5\%). The smaller panel in the upper right corner shows the $u-v$ plane.

Table 1. Calibrators used for the observations of $\eta$ Ser.

\begin{tabular}{lccccc}
\hline \hline Star & $m_{V}$ & $m_{K \mathrm{~s}}$ & Spect. & $\begin{array}{c}\theta_{\mathrm{UD}(\mathrm{K})} \\
(\mathrm{mas})\end{array}$ & $\begin{array}{c}\gamma \\
\left({ }^{\circ}\right)\end{array}$ \\
\hline IRC+00339 & 5.5 & 2.6 & K2III & $1.402 \pm 0.018$ & 6.8 \\
HR 6650 & 6.2 & 3.2 & K1III-IV & $1.123 \pm 0.015$ & 16.7 \\
HR 7809 & 6.1 & 3.6 & K1III & $1.055 \pm 0.015$ & 31.1 \\
\hline
\end{tabular}

Notes. $m_{v}, m_{K \mathrm{~S}}$ : magnitudes in $V$ and $K$ bands; $\mathrm{UD}(\mathrm{K})$ : uniform disk angular diameter; $\gamma$ : angular distance to $\eta$ Ser.

Kervella et al. 2004a; Mérand et al. 2006a). For the reported observations, we used the CHARA baselines S2-W2 and E2-S1, with ground lengths of 177 and $279 \mathrm{~m}$, respectively. The calibrator stars were chosen from the catalogue compiled by Mérand et al. (2005), using criteria defined by these authors (Table 1). They were observed immediately before and/or after $\eta$ Ser in order to monitor the interferometric transfer function of the instrument.

All our results were obtained using visibility models that take into account bandwith smearing, as described in Aufdenberg et al. (2006). Since the optical filter for FLUOR is quite wide, it is extremely important to take band width smearing in account, especially if one collects data near a visibility null where the visibility varies substantially inside the waveband. We shall not fully describe this method in this paper, as it is very specific to the instrument and filter details. These details are available, however, directly from the authors. For visual comparison, we plot in Fig. 1 the monochromatic visibility for $\lambda=2.16$ microns.
Table 2. Calibrated squared visibility measurements of $\eta$ Ser.

\begin{tabular}{lcccc}
\hline \hline MJD & $\begin{array}{c}B \\
(\mathrm{~m})\end{array}$ & $\begin{array}{c}\text { PA } \\
\left({ }^{\circ}\right)\end{array}$ & $\begin{array}{c}V^{2} \pm \sigma \\
(\%)\end{array}$ & L1 \\
\hline 53930.377 & 214.68 & -7.77 & $0.00715 \pm 0.00041$ & \\
53930.400 & 219.44 & -13.90 & $0.00781 \pm 0.00020$ & \\
53936.364 & 171.46 & -38.16 & $0.00924 \pm 0.00053$ & $\bullet$ \\
53937.302 & 157.44 & -32.36 & $0.02710 \pm 0.00114$ & $\bullet$ \\
53937.320 & 162.43 & -34.71 & $0.01978 \pm 0.00093$ & $\bullet$ \\
53937.338 & 166.67 & -36.46 & $0.01272 \pm 0.00065$ & $\bullet$ \\
\hline
\end{tabular}

Notes. MJD: modified Julian date of the observation; $B$ : projected baseline length; PA: azimuth of the projected baseline (counted positively from North to East); $V^{2}$ : squared visibility; L1: "•" means measurements obtained in the first lobe of the visibility.

Table 3. Results of the fits to the data for different models.

\begin{tabular}{lcccccc}
\hline \hline Model & \multirow{2}{*}{$\begin{array}{c}\mathrm{LD} \\
\text { (mas) }\end{array}$} & $\alpha$ & \multicolumn{2}{c}{ First lobe } & \multicolumn{2}{c}{ All } \\
& & & $\chi^{2}$ & $P$ & $\chi^{2}$ & $P$ \\
\hline Uniform disk & $2.855 \pm 0.007$ & 0.000 & 1.89 & 1 & 17.1 & 0 \\
ATLAS9 & $2.936 \pm 0.007$ & 0.176 & 1.59 & 1 & 2.2 & 0 \\
MARCS-pp & $2.940 \pm 0.007$ & 0.183 & 1.58 & 1 & 2.1 & 0 \\
PHOENIX & $2.944 \pm 0.007$ & 0.196 & 1.57 & 1 & 1.9 & 0 \\
MARCS-sph & $2.945 \pm 0.007$ & 0.193 & 1.56 & 1 & 1.9 & 0 \\
adjusted LD & $2.958 \pm 0.027$ & $0.23 \pm 0.05$ & 1.52 & 0 & 2.1 & 2 \\
\hline
\end{tabular}

Notes. $\theta_{\mathrm{LD}}$ : CLD corrected diameter; $\alpha$ : equivalent power law parameter; $\chi^{2}$ and $P$ : reduced $\chi^{2}$ and number of parameters fitted (if $P=0$, the $\chi^{2}$ is only computed). "first lobe" referes to data marked with a "•" in Table 2.

\section{Angular diameter}

\subsection{Limb darkening model comparison}

In order to estimate the angular diameter from the measured visibilities (Table 2) it is necessary to know the intensity distribution of the light on the stellar disk, that is the limb darkening (LD). In this Section, we present the results of two approaches based on an assumed intensity distribution:

1. adjust a simple uniform disk model to the $V^{2}$ measurements. The uniform disk (UD) model, although unphysical, is a useful tool for interferometry when the stars are marginally resolved since it is a single parameter model;

2. assume a limb darkened profile from existing atmosphere models. Here we use the stellar parameters derived recently for $\eta$ Ser by Hekker \& Meléndez (2007): $T_{\text {eff }}=4955 \mathrm{~K}, \log g=3.20,[\mathrm{Fe} / \mathrm{H}]=-0.21, V_{\text {turb }}=$ $3.52 \mathrm{~km} \mathrm{~s}^{-1}$. For the computation of the intensity profile of the star, three models are used: the classical ATLAS9 and PHOENIX models, approximated using Claret's (2000) four-parameter laws in the $K$ band, and the MARCS model (Gustafsson et al. 2008) ${ }^{1}$, using intensities computed using TURBOSPECTRUM (Alvarez \& Plez 1998), with spherical ("-sph") and plane-parallel geometries ("-pp”).

The results of the fits are presented in Table 3. In each case, the angular diameter of the star is calculated by fitting, using a $c h i^{2}$ minimization, only the four $V^{2}$ measurements obtained in the first lobe of the visibility function, where our measurements are insensitive to limb darkening. We then compute the reduced $\chi^{2}$ using all the $V^{2}$ measurements which gives us a metric of the

1 http://www.marcs.astro.uu.se/ 
quality of the limb darkening prediction of each type of model. In each case, the quoted error bar on $\theta_{\mathrm{LD}}$ includes the statistical uncertainty, the systematic uncertainties from the effective instrumental spectral bandpass, and the uncertainty in calibrator angular diameters. The reader is referred to Perrin (2003) for a detailed description of the error propagation method we used.

Note that the presence of circum-stellar material might bias the angular diameter estimates. We will investigate this possibility in Sect. 3.5.

\subsection{Parametrized power-law limb darkening fit}

As shown in Fig. 1, two of the data points were obtained in the second lobe of the visibility function. The star is therefore fully resolved by the interferometer, and these data can be used to investigate the limb darkening, relatively independently from the angular diameter estimate. However, as there are only two $V^{2}$ points, we cannot measure the actual shape of the intensity distribution. Hestroffer (1997) showed that a power law $I_{\lambda}(\mu) / I(1)=\mu^{\alpha}$ (with $\alpha \geq 0$ and $\mu=\cos (\theta)$ ) is a good approximation to stellar limb darkening profiles. Such an approximation presents two advantages: it requires only one more free parameter compared to a simple angular diameter fit (the $\alpha$ exponent); and it gives an analytical expression of the visibility function, thus simplifying the visibility curve modeling. By definition, this model leads to a stellar radius defined as $\mu=0$. For $\eta$ Ser, the two-parameter model fitting gives:

$\alpha=0.23 \pm 0.05( \pm 25 \%)$

$\theta_{\mathrm{LD}, \alpha}=2.958 \pm 0.027 \operatorname{mas}( \pm 0.9 \%)$.

The two parameters in this fit are correlated, and so the derived value of $\theta_{\mathrm{LD}}$ is less precise than the single-parameter based on the points in the first lobe, but it is statistically consistent.

In Fig. 1, we did not overplot the ATLAS9, PHOENIX and both MARCS models because at the scale of this plot, they would overlap.

\subsection{Comparison between power law CLD and photospheric models}

It is important to understand the limitations of the power-law fitting performed above. We do not claim that the star actually has a power law CLD profile. However, the visibility profiles of the other models can be approximated by the power law CLD. The visibility of the ATLAS9 model corresponds to the visibility given by a power law $\alpha=0.176$, the MARCS-pp model gives $\alpha=0.183$. Conversely, the spherical models PHOENIX and MARCS-sph correspond to $\alpha=0.196$ and $\alpha=0.193$ respectively. In other words, our fitted value of $\alpha$ is statistically compatible with the ATLAS9, PHOENIX and both MARCS models and at this level of precision, the data do not allow us to distinguish between the models. It does however, seem that models using the spherical geometry (PHOENIX and MARCS-sph) give slightly better agreement than models using the plane-parallel geometry (ATLAS9 and MARCS-pp).

\subsection{Limb darkened disk angular (unbiased) diameter}

Based on the results of the comparison in setout in Sect. 3.1 and our two-parameter fit in Sect. 3.2, the best estimate of the photospheric angular size of $\eta$ Ser was choosen to be the value given by the spherical models (an average of PHOENIX and MARCS$\mathrm{sph}$ ). This corresponds to a fit on all the data set with a power law of $\alpha=0.1945$ resulting in

$\theta_{\mathrm{LD}}=2.944 \pm 0.006 \operatorname{mas}( \pm 0.2 \%)$,

with a reduced $\chi^{2}$ of 1.9 . The error bar only takes into account the statistical errors coming from the visibility measurements and the on-sky calibration. At this level of precision, one should also take into account the precision of the FLUOR transmission calibration which is estimated to be of the order of $0.01 \mu \mathrm{m}$. We computed the predicted visibilities of the calibrators and fitted the diameters with this spectral shift in the instrumental model. This lead to final shift of $0.27 \%$ in the estimated angular diameters of $\eta$ Ser, or 0.008 mas. Hence, adding this error quadratically to the statistical error, we estimate the final diameter of $\eta$ Ser to be:

$\theta_{\mathrm{LD}}=2.944 \pm 0.010$ mas $( \pm 0.34 \%)$.

\subsection{On the possibility of the presence photometric excess}

Another source of bias of the interferometric measurement of angular diameters is the presence of fully resolved circumstellar material, being seen as a photometric excess. This will lead to a measured visibility apparently not converging to unity as the baseline decreases. We tested this possibility with a fit of the diameter and such excess in the first lobe which lead to $\theta_{\mathrm{LD}}=$ $2.92 \pm 0.04$ mas with an uncorrelated flux of $9 \pm 8 \%$ of stellar flux. In the second lobe, this leads to $\theta_{\mathrm{LD}}=2.940 \pm 0.006$ mas with an uncorrelated flux of $1.1 \pm 1.2 \%$. In other words, our first lobe data are not very sensitive to the effect because we are close to the first null and one needs measurements at very short baselines, as discussed in Mérand et al. (2006b). On the other hand, including the second lobe data rules out the presence of such a bias, at the $1 \%$ level in terms of photometric precision, and we are confident that even in the presence of a photometric bias of $1 \%$, our final angular diameter estimate remains unbiased.

\section{Linear radius and discussion}

The comparison of our different fits to the data points in the second lobe showed that the limb darkening power law is in good agreement with the photospheric models. As a comparison with the angular diameter values derived in Sect. 3, $\eta$ Ser is present in Bordé et al.'s (2002) catalogue of interferometric calibrator stars, where its angular diameter was estimated from its spectral energy distribution to be $\theta_{\mathrm{LD}}=2.98 \pm 0.032$ mas, within $1.1 \sigma$ of our measurement, but three times less precise. Considering the $V$ and $K$ magnitudes of $\eta$ Ser of $m_{V}=3.25 \pm 0.02$ and $m_{K}=1.05 \pm 0.02$ (Morel \& Magnenat 1978, with typical uncertainties), the $[V, V-K]$ surface brightness-color relation calibrated by Kervella et al. (2004b) predicts an angular diameter of $\theta_{\mathrm{LD}}=2.975 \pm 0.054$ mas, in excellent agreement with our CHARA/FLUOR measurement. Finally, the spectral energy distribution fitting code developed for the work presented in Kervella et al. (2009) based on photospheric modeling of wide band photometric measurements $(B, V, J, H, K)$, gives an angular diameter of $2.935 \pm 0.050$ mas and an effective temperature of $4925 \pm 40 \mathrm{~K}$.

The linear radius of $\eta$ Ser, can be calculated based on a parallax from the reprocessed Hipparcos catalogue by van Leeuwen $(2007 \mathrm{a}, \mathrm{b})$ of $\pi(\eta$ Ser $)=53.94 \pm 0.18 \mathrm{mas}( \pm 0.33 \%)$. This value, while more precise, is in good agreement with, 
van Altena et al. (1995) and the initial Hipparcos value (ESA 1997). The photospheric linear radius is therefore

$R(\eta$ Ser $)=5.897 \pm 0.028 R_{\odot}( \pm 0.47 \%)$.

\section{Conclusion}

Our results show that the limb darkening of $\eta$ Ser is modeled well by the existing spherical models PHOENIX and MARCS, and to a smaller extent by plane-parallel ATLAS. This reinforces the already widely accepted belief that for simple case such as non-pulsating stars, photospheric models can be used to debias an interferometrically determined angular diameter. In particular, it confirms that the interferometric calibrator catalogues from Bordé et al. (2002) and Mérand et al. (2005) are not affected by a systematic bias due to the assumed limb darkening model. They contain mostly $\mathrm{K}$ giants of similar spectral types as $\eta$ Ser. The derived radius of $\eta \operatorname{Ser}\left(5.897 \pm 0.028 R_{\odot}\right)$ is among the most accurate of such measurements available, and will be a valuable constraint for the modeling of this star when combined with asteroseismic data, as demonstrated by our measurement of the diameter of a G giant, $\epsilon$ Oph, and the successful modeling of its detected asteroseismic frequencies (Mazumdar et al. 2009).

Acknowledgements. The authors would like to thank the CHARA Array and Mount Wilson Observatory day-time and night-time technical and administrative staff for their support. The CHARA Array was constructed with funding from Georgia State University, the National Science Foundation, the W. M. Keck Foundation, and the David and Lucile Packard Foundation. The CHARA Array is operated by Georgia State University with support from the College of Arts and Sciences, and from the National Science Foundation under NSF Grant AST 0606958. This work also received the support of PHASE, the high angular resolution partnership between ONERA, Observatoire de Paris, CNRS and University Denis Diderot Paris 7. This research took advantage of the SIMBAD and VIZIER databases at the CDS, Strasbourg (France), and NASA's Astrophysics Data System Bibliographic Services.

\section{References}

Alvarez, R., \& Plez, B. 1998, A\&A, 330, 1109

Aufdenberg, J. P., Mérand, A., \& Coudé du Foresto, V. 2006, ApJ, 645, 664; Erratum 2006, ApJ, 651, 617

Barban, C., de Ridder, J., Mazumdar, A., et al. 2004, Proc. SOHO 14/GONG 2004 Workshop (ESA SP-559), Helio- and Asteroseismology: Towards a Golden Future, 12-16 July, New Haven, Connecticut, USA, ed. D. Danesy, 113

Bordé, P., Coudé du Foresto, V., Chagnon, G., \& Perrin, G. 2002, A\&A, 393, 183

Claret, A. 2000, A\&A, 363, 1081

Coudé du Foresto, V., Ridgway, S., \& Mariotti, J.-M. 1997, A\&AS, 121, 379

Coudé du Foresto, V., Bordé, P., Mérand, A., et al. 2003, Proc. SPIE, 4838, 280

Creevey, O. L., Monteiro, M. J. P. F. G., Metcalfe, T. S., et al. 2007, ApJ, 659, 616

Cunha, M. S., Aerts, C., Christensen-Dalsgaard, J., et al. 2007, A\&AR, 14, 217

ESA 1997, The Hipparcos and Tycho Catalogues, ESA SP-1200

Gustafsson, B., Edvardsson B., Eriksson K., et al. 2008, A\&A, 486, 951

Hekker, S., \& Meléndez, J. 2007, A\&A, 475, 1003

Hekker, S., Aerts, C., De Ridder, J., \& Carrier, F. 2006, A\&A, 458, 931

Hestroffer, D. 1997, A\&A, 327, 199

Kervella, P., Ségransan, D., \& Coudé du Foresto, V. 2004a, A\&A, 425, 1161

Kervella, P., Thévenin F., Di Folco, E., \& Ségransan, D. 2004b, A\&A, 426, 297, K04

Kervella, P., Mérand, A., \& Gallenne, A. 2009, A\&A, 498, 425

Mazumdar, A., Merand, A., Demarque, P., et al. 2009, A\&A, 503, 521

Mérand, A., Bordé, P., \& Coudé du Foresto, V. 2005, A\&A, 433, 1155

Mérand, A., Coudé du Foresto, V., Kellerer, A., et al. 2006a, Proc. SPIE, 6268, 46

Mérand, A., Kervella, P., Coudé Du Foresto, V., et al. 2006a, A\&A, 453, 155

Morel, M., \& Magnenat, P. 1978, A\&AS, 34, 477

Perrin, G. 2003, A\&A, 400, 1173

ten Brummelaar, T. A., McAlister, H. A., Ridgway, S. T., et al. 2005, ApJ, 628, 453

van Altena, W. F., Lee, J. T., \& Hoffleit, E. D. 1995, The General Catalogue of Trigonometric Stellar Parallaxes, 4th edition, Yale University Observatory van Leeuwen, F. 2007a, Hipparcos, the New Reduction of the Raw Data, Astrophysics and Space Science Library, Vol. 350 (Springer)

van Leeuwen, F. 2007b, A\&A, 474, 653 